\title{
Estrategias participativas y transmedia de empoderamiento ciudadano. El caso de San Juan de Ocotán
}

\author{
Afra Citlalli Mejía Lara \\ Instituto Tecnológico y de Estudios Superiores \\ de Occidente, México \\ afra@iteso.mx \\ https://orcid.org/0000-0002-4405-0179
}

\author{
Diego Zavala Scherer \\ Tecnológico de Monterrey, \\ México \\ diego.zavala@tec.mx \\ https://orcid.org/0000-0002-7362-4709
}

\section{Participatory and transmedia strategies for citizen empowerment: San Juan de Ocotán as a Case Study}

\begin{abstract}
RESUMEN ABSTRACT
El artículo presenta el proyecto participativo desarrollado en San Juan de Ocotán durante un año, para crear un grupo de producción de medios con jóvenes residentes del área.

Se utilizaron metodologías colaborativas donde los par-

ticipantes se organizaron en tres equipos, y produjeron aproximadamente dos horas de piezas documentales, una exposición fotográfica y un sitio web que alberga todos los demás productos. Este proyecto transmedia funciona como

primera fase del desarrollo de repositorio digital para la creación de una memoria colectiva del barrio. Tras la presentación pública de resultados de los talleres en el centro cultural de San Juan, poco más de diez jóvenes decidieron continuar con el trabajo de hacer transferencia tecnológica y procesos participativos en la comunidad y se constituyeron como una compañía de producción audiovisual a la que llamaron Documachete. Ahora desarrollan un largometraje documental sobre la fiesta del barrio, y planean otras pro-

This paper presents the participatory project developed in San Juan de Ocotán through a whole year to bring together a media production group with the youngsters living in the area. Using collaborative methodologies, the participants organized into three teams, and they developed approximately two hours of documentary productions, a photographic exposition, and a website to upload all their products. This transmedia webpage works as the first phase of digital collective memory for the neighborhood. After the public presentation of the results in the cultural center at San Juan, ten young men and women of the group decided to continue the work of technological transference and participatory processes in the neighborhood and constitute a media production Company called Documachete. Now they are developing a long feature documentary about the main festivity of the community and planning other media projects needed in San Juan.
\end{abstract} ducciones basadas en las necesidades mediáticas de San Juan.

PALABRAS CLAVE KEYWORDS

Proyecto participativo; Literacidades transmedia; Memoria digital; San Juan de Ocotán.

Participatory project; Transmedia literacies; Digital memory; San Juan de Ocotán. 


\section{Introducción}

Dentro del panorama del giro digital y los cambios que ha traído la llamada cultura de la convergencia (Jenkins, 2008), este artículo se pregunta cómo suceden estas integraciones de tecnologías en ambientes educativos no escolarizados, en general, y cómo se pueden vincular al desarrollo comunitario, en particular.

A partir de esta preocupación sobre los cambios tecnológicos y de integración a comunidades de aprendizaje diversas fuera de los espacios académicos clásicos, es que nace el proyecto de talleres de producción audiovisual y transmedia en el pueblo urbano de San Juan de Ocotán, el cual servirá como estudio de caso en este contexto. Por las condiciones de la comunidad - que luego detallaremos- las preguntas deben ser matizadas, pues a pesar de que encontramos presencia de muchos de los avances tecnológicos del giro digital (internet, teléfonos celulares, computadoras), lo cierto es que también existe una gran brecha digital que considerar.

Si como señala Carlos Scolari, "la convergencia es el caldo de cultivo donde nacen las narrativas transmedia" (2013, p. 63), es fundamental establecer cómo sucede esta convergencia en situaciones y contextos específicos. En nuestro caso, el proyecto sucede en una población estigmatizada como violenta y con unas características sociodemográficas muy específicas que representan un reto para considerarla como ideal para el cultivo de proyectos transmediales. Aun así, el espacio resulta ideal para proponer las posibilidades de desarrollo de proyectos de medios, justo por las carencias que la intervención tuvo.

Y sobre este caso, lo que se pretende mostrar es cómo la práctica de producción colaborativa audiovisual, y su forma final como una página web, se convirtió en una narrativa, en una memoria del barrio, y lo más importante: en un colectivo de jóvenes que encontró en el taller y las acciones asociadas a él, un mecanismo de participación dentro de su comunidad.

\section{Marco teórico y conceptual}

La propuesta de investigación que sustenta el presente trabajo parte de la intersección de la producción mediática con la educación. Explora los medios en el contexto digital y sus funciones dentro de la sociedad contemporánea como herramienta de aprendizaje, o bien, desarrollo de competencias. Nos interesa, particularmente, establecer estas literacidades o alfabetidades como mecanismos potenciales para generar actos de habla en la tradición de Austin (2020) y Searle (1969), como se explicará en el siguiente apartado dedicado al caso. La intención desde el principio era llevar a la práctica, en el proyecto de base comunitaria que finalmente se desarrolló en San Juan de Ocotán, Jalisco, México, esta idea de la construcción de sentido a través de un trabajo de producción mediática colaborativa que permitiera hacer trabajo de investigación-ac- ción (Colmenares, 2012) sobre las literacidades digitales de jóvenes del barrio. Era, por supuesto, también, una intervención en un espacio urbano hipercodificado como peligroso, donde la educación pareciera más un privilegio que una opción para los adolescentes y adultos jóvenes.

Las condiciones, por tanto, invitaban mucho más a pensar en el cosmos de la transferencia de medios, el trabajo asambleario y participativo, que a llevar los medios a un espacio de educación formal para ver si la presencia de los medios de producción de imágenes y sonidos de base digital modificaban las prácticas o permitía esta apropiación medial, multimedial o transmedial por parte de los jóvenes. En ese sentido, por supuesto que los trabajos de Alejandro Piscitelli y el grupo de investigadores de El proyecto Facebook y la posuniversidad (Piscitelli, Adaime y Binder, 2010), y el proyecto Transmedia literacy de Carlos Scolari (2017), sobre alfabetidades transmediales, sirven como referentes poderosos sobre cómo intervenir, categorizar e interpretar la disrupción que significa para la educación la presencia de los medios y las redes sociodigitales en sus procesos. En nuestro caso, servirían sólo de guías metodológicas limitadas o matizadas por el contexto de intervención y las dinámicas generadas en el espacio público y con asistentes voluntarios.

De igual manera, al decidir que el proyecto sería en un contexto no formal y que los procesos de enseñanza, transferencia o co-creación se darían en espacios negociados de encuentro, el foco se desplaza de forma orgánica hacia la inclusión de las necesidades e inquietudes temáticas y sociales de los participantes. De esta manera, no sólo se pretendía capacitarlos en la producción audiovisual y digital, sino también incluir sus preocupaciones, problemáticas y temáticas como parte del impacto social que el proyecto proponía.

En este tenor, y debido a las necesidades de los jóvenes y adultos que se integraron a los talleres, fue necesario incluir la dimensión transmedial o la base digital a manera de repositorio de los contenidos generados. Los talleres giraron alrededor de temas como la estigmatización, la identidad, la relación personal con el barrio, la migración y la propia experiencia de ser joven en San Juan de Ocotán. De esta manera, el ciberespacio potenciaba la capacidad de preservar y compartir lo producido y aprendido. Por ello, al pensar la condición transmedia debemos considerar dos dimensiones, más allá de las literacidades o alfabetidades que el hacer una página web o blog colaborativo reporta.

Nos parece que esto nos trasladó a otro territorio transmedial, o si se prefiere, a una expansión. Creemos que la manera de pensar el transmedia que define de manera más precisa el proyecto narrado en el siguiente apartado es el de Ute Seydel (2018), siguiendo a Astrid Erll (2008). La sistemática representación del registro mediático de barrios - como San Juan de Ocotán - como espacios de violencia y problemas sociales es solo una parte de la memoria colectiva de la comunidad. Y 
en ese sentido, la producción realizada en los talleres ayuda a visiblizar las tensiones de lo que las autoras denominan como memoria transmedial.

Crear un archivo fotográfico, videográfico, sonoro e interactivo del barrio en un momento específico muestra la intermedialidad del proyecto y sus aspiraciones a explorar, así como explotar las potencialidades que cada medio ofrece a los jóvenes para plasmar su cultura, identidad, deseos y preocupaciones. Nos enfrentamos a un intento de creación de memoria cultural desde una posición inusual, donde los jóvenes no solo aportan registros privados que pudieran ser compartidos en alguna plataforma como YouTube; en este caso, al salir de forma consciente a registrar la vida del barrio, están negociando la forma material y discursiva de estos mensajes, que también sirven como premediaciones y precedente de registro para el futuro del colectivo. $Y$ justo este componente es el que permite dislocar la estereotipación que los medios informativos y comerciales hacen de sitios como San Juan de Ocotán.

Esta irrupción de los jóvenes habitantes de la comunidad en el sistema mediático de representaciones de su barrio se corresponde con aquello que los casos alrededor del mundo relatan sobre la construcción de agencia y acción cívica de los participantes en este tipo de proyectos; tanto en el sentido amplio sobre la perspectiva de cómo se inserta la producción mediática en la acción social y política de los ciudadanos (Artz, 2020; Ravenscroft, 2020; Wyatt, et al., 2013), como en las experiencias particulares del trabajo fílmico alrededor de la creación de conciencia, identidad, soberanía de las comunidades y agencia (Dedemaine, Marcoux y St. Amand, 2020; Gumucio, 2014; Mejía, 2017; Zavala y Leetoy, 2016).

Finalmente, como dice Johnson (2013, p. 2): "una de las nuevas dimensiones del entretenimiento transmedia contemporáneo es nuestro reconocimiento de la transmedia en cuanto tal". Aunque este texto no trata forzosamente de narrativas transmedia de entretenimiento, esta participación activa en la creación por parte de los habitantes de San Juan puede ser vista como una forma de conciencia emergente, así como de productores audiovisuales que están generando una propuesta de lectura e identificación de la comunidad: reconocen y se reconocen haciendo transmedia.

Esta forma de conciencia vinculada a las acciones que acompañan la literacidad mediática e interactiva es claramente equiparable a la performatividad que acompaña la dimensión lingüística de los actos de habla. Esta noción que también empata poderosamente con la tradición documental que se cuestiona sobre la relación del cuerpo de quien filma, de quien es filmado y de la cámara que interactúa entre ellos, o también se puede comparar al espíritu del espectador televisivo que Jason Mittell relata en su libro Complex TV (2015) al referirse a cómo el consumidor televisivo de series reconoce la estética operacional y distingue lo que la narrativa espera de él o ella.
Lo que nos parece relevante y fértil del proyecto es acompañar a un grupo de jóvenes a poscionarse en el centro del proceso de creación y comunicación para cuestionarse qué quieren decir y qué le quieren proponer a quien mire sus producciones.

\section{Caso de estudio y contexto de investigación}

La experiencia que ahora relatamos surge, como habíamos avanzado, con la forma de un proyecto de investigación-acción en el pueblo de San Juan de Ocotán; un pueblo originario cuyos primeros registros datan de la llegada de los españoles, y que actualmente es parte de la periferia de la ciudad de Guadalajara, la segunda ciudad más grande de México. Su vecindad con Guadalajara permite que sus pobladores puedan acceder a las fuentes de trabajo que ofrece la urbe, pero esto también ha tenido como consecuencia un permanente despojo de su territorio y una constante violencia real y simbólica hacia su forma de vida. Esta condición se ha recrudecido en los últimos años con el acelerado crecimiento de la ciudad, ya que actualmente San Juan de Ocotán se encuentra en la zona de mayor plusvalor de la ciudad, rodeada por industrias, fraccionamientos exclusivos, campos de golf, tres de las avenidas de tránsito pesado más importantes de la ciudad y la vía del tren cuya ruta utilizan los migrantes mexicanos y centroamericanos que se van hacia el norte del país para intentar cruzar ilegalmente la frontera. Todo ello hace que San Juan de Ocotán tenga una realidad geopolítica muy compleja.

Por otro lado, aunque a lo largo de los años San Juan de Ocotán ha perdido su identidad como pueblo indígena, todavía mantiene muchas de sus tradiciones, en las cuales se observan huellas de su historia, desde la época de la conquista. La vida y los ritmos del pueblo se encuentran marcados por el calendario litúrgico y las fiestas patronales. Uno de los hallazgos de esta investigación, sobre los cuales no vamos a profundizar en esta ocasión, fue que la Fiesta de los tastuanes, dedicada al Santo Santiago - la fiesta más importante del pueblo -, además de ser una fiesta sobre la conquista española, ha sido resignificada como un mecanismo de resistencia de su lucha contra el despojo histórico de su territorio y los procesos de urbanización de los últimos años. Es así como San Juan de Ocotan se sitúa en una zona fronteriza en permanente tensión entre la conservación de su forma de vida y sus tradiciones y la integración a la vida urbana moderna.

El proyecto que vamos a describir en este trabajo consiste en un proceso de producción audiovisual colaborativa con los jóvenes del barrio y la generación de un documental web, cuyo objetivo era el de construir un espacio de memoria del barrio en forma de un acervo audiovisual. Este proceso formó parte del trabajo de campo de la tesis doctoral de Afra Citlalli Mejía Lara', cuya pregunta inicial se situaba en la generación de procesos de autorrepresentación audiovisual producidos por los mismos habitantes del barrio, los cuales pudieran ser confrontados con los discursos mediáticos que tienden a representar 


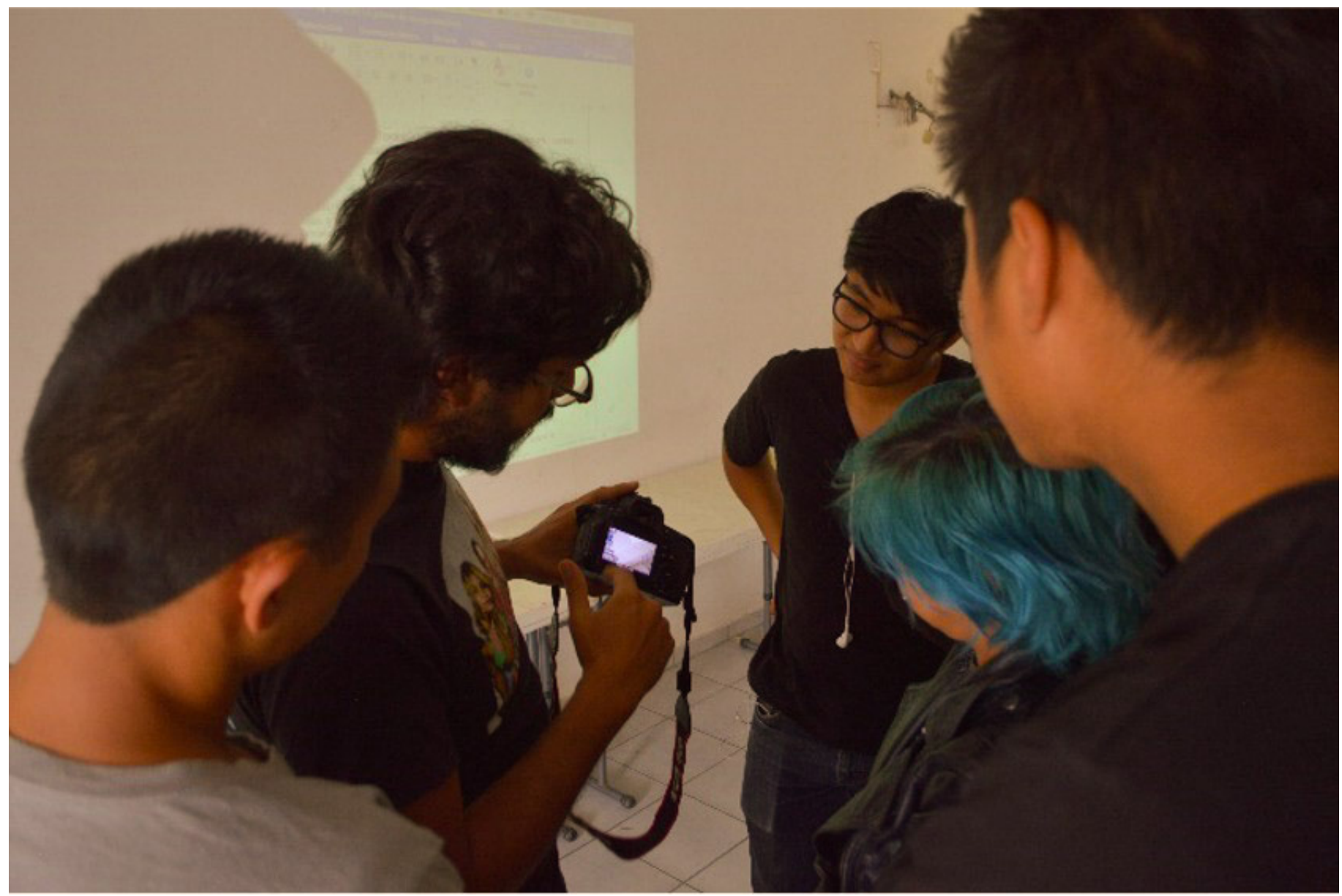

Figura 1. Taller de cine comunitario. Fuente: elaboración propia.

a San Juan de Ocotán únicamente como un barrio marginal y con altos índices de violencia, de la periferia de Guadalajara. Sin embargo, a lo largo del trabajo surgieron otras preguntas relacionadas con las alfabetidades tecnológicas y digitales, las cuales no pueden ser desligadas del contexto histórico y cultural del barrio.

Una de los retos de este trabajo fue utilizar el audiovisual como una herramienta de habla de los sujetos subalternos, en términos de Spivak. En su texto "¿Puede hablar el subalterno?", Chakravorty Spivak recuperará a Brue Tiebout McCuily (1940) quien en su libro English Education and the Origin of Indian Nationalism describe cómo durante el proceso colonial en la India, la educación se convirtió en un instrumento de silenciamiento de la cultura india, ya que uno de sus objetivos era el de "formar una clase que pueda funcionar como intérprete entre nosotros y los millones a quienes gobernamos (...) que sean indios en sangre y color pero ingleses en gusto" (Spivak, 1988, p. 14). A su vez, esta educación tuvo como consecuencia reducir todo a la escritura, con lo cual otras formas de expresión se invisiblizaron. Los únicos sujetos con acceso al habla eran aquellos nativos letrados, educados para coincidir con los intereses de los colonos, que a su vez se convirtieron en las fuentes de los intelectuales europeos, quienes no tenían acceso a las grandes masas de iletrados. Es por ello que Spivak concluye que los sujetos subalternos no tienen voz y que no pueden ser escuchados, especialmente por las élites intelectuales de occidente. Sin embargo, este silenciamiento en realidad no es una falta de habla, sino como la misma Spivak explica, es una sordera por parte de las metrópolis del poder y del conocimiento occidentales incapaces de ver y escuchar lenguajes y culturas diferentes. Es por ello que el proyecto propuesto buscaba empoderar y hacer más audible el habla de los jóvenes de San Juan de Ocotán.

Por otro lado, la apuesta por el uso del audiovisual tiene que ver con un intento de descentrar al conocimiento logocéntrico sustentado en la razón y en la palabra. Uno de los pilares de la modernidad ha sido el instaurar un modo de conocer como la única forma de conocimiento válido: la razón. Diversos teóricos poscoloniales han dado cuenta de cómo esta hegemonía epistemológica no sólo ha tenido como consecuencia una desvalorización de otras formas de conocimiento, como la filosofía y el arte, sino que también ha operado como una forma de discriminación hacia otras culturas señaladas como "atrasadas" o "salvajes". La dificultad que tienen algunas comunidades para adaptarse a las estructuras escolarizadas y a las formas logocéntricas de conocimiento no está únicamente relacionada con su falta de acceso, para ello también se deben tomar en cuenta que no todas las culturas comparten la 
misma matriz de conocimiento y que, por ello, la escuela también se puede volver un espacio de discriminación social.

En el caso de San Juan de Ocotán la deserción escolar es común entre sus habitantes. De acuerdo al censo de 2010, mientras en el municipio de Zapopan donde se encuentra el pueblo, el promedio de escolaridad es de 10 años, en San Juan de Ocotán es de 8 años cursados. A pesar de que la escuela es valorada positivamente y los padres desean que sus hijos continúen en la escuela, esta no es valorada como un factor de ascenso económico. Uno de los argumentos para la deserción es que la escuela no les iba a garantizar un futuro mejor, ya que "de todos modos no hay trabajo". En entrevistas con algunos de ellos, mencionaron que dejaban la escuela porque tenían necesidad de trabajar, o porque requería ingresos inmediatos, o porque se casaban jóvenes y se veían obligados a mantener a sus hijos. Sin embargo, casi todos también describieron la experiencia escolar como difícil, "aburrida" y en la que sufrieron algún tipo de discriminación, ya fuera porque se les dificultaba el aprendizaje, o por su color de piel, o por su forma de hablar (Mejía Lara, 2013. pp.152-153).

En el mundo moderno, la falta de dominio de la matriz de conocimiento sustentada en la lectoescritura y la razón, se convierte en una condición de exclusión y de marginalidad, pero también se traduce en un silenciamiento de su capacidad de enunciación. Así, los habitantes de San Juan de Ocotán se han visto históricamente excluidos de la posibilidad de autorrepresentarse en los relatos históricos o periodísticos y muchos de ellos tienden a subrayar su condición de "marginales", "salvajes" y "atrasados". Como ejemplo, en una primera etapa de la investigación, Afra Mejía realizó un seguimiento de notas de periódico que aparecieron en las alertas de Google de abril de 2011 al 13 de octubre de 2013, las cuales dieron como resultado 74 notas, de las que el 70 \% aparecía en la nota roja y prácticamente el único discurso sobre San Juan de Ocotán era el de un pueblo marginal y violento de la periferia de Guadalajara (Mejía Lara, 2013). Es por ello que Mejía Lara apostó por un lenguaje donde lo sensorial, lo performativo y lo emocional pudieran tener primacía como forma de habla por encima del lenguaje escrito.

Es en este contexto se desarrolló un Taller de cine comunitario (Figura 1), el cual se llevó a cabo todos los sábados durante casi un año en colaboración con el colectivo de cineastas Documotora $^{2}$ donde se procuró recuperar su experiencia, tanto en el trabajo de barrio, como en la producción de documentales colaborativos. Durante el Taller, al mismo tiempo que los jóvenes adquirían habilidades técnicas y narrativas, se iban generando procesos de producción colaborativa como autorretratos y retratos fotográficos, cineminutos sobre lugares que les fueran significativos, cortometrajes breves sobre su propia experiencia de la Fiesta de los tastuanes, etcétera. Los productos finales de este proceso fueron tres cortometrajes colaborativos de aproximadamente media hora basados en intereses o preocupaciones de los mismos jóvenes participantes del Taller (Figura2)

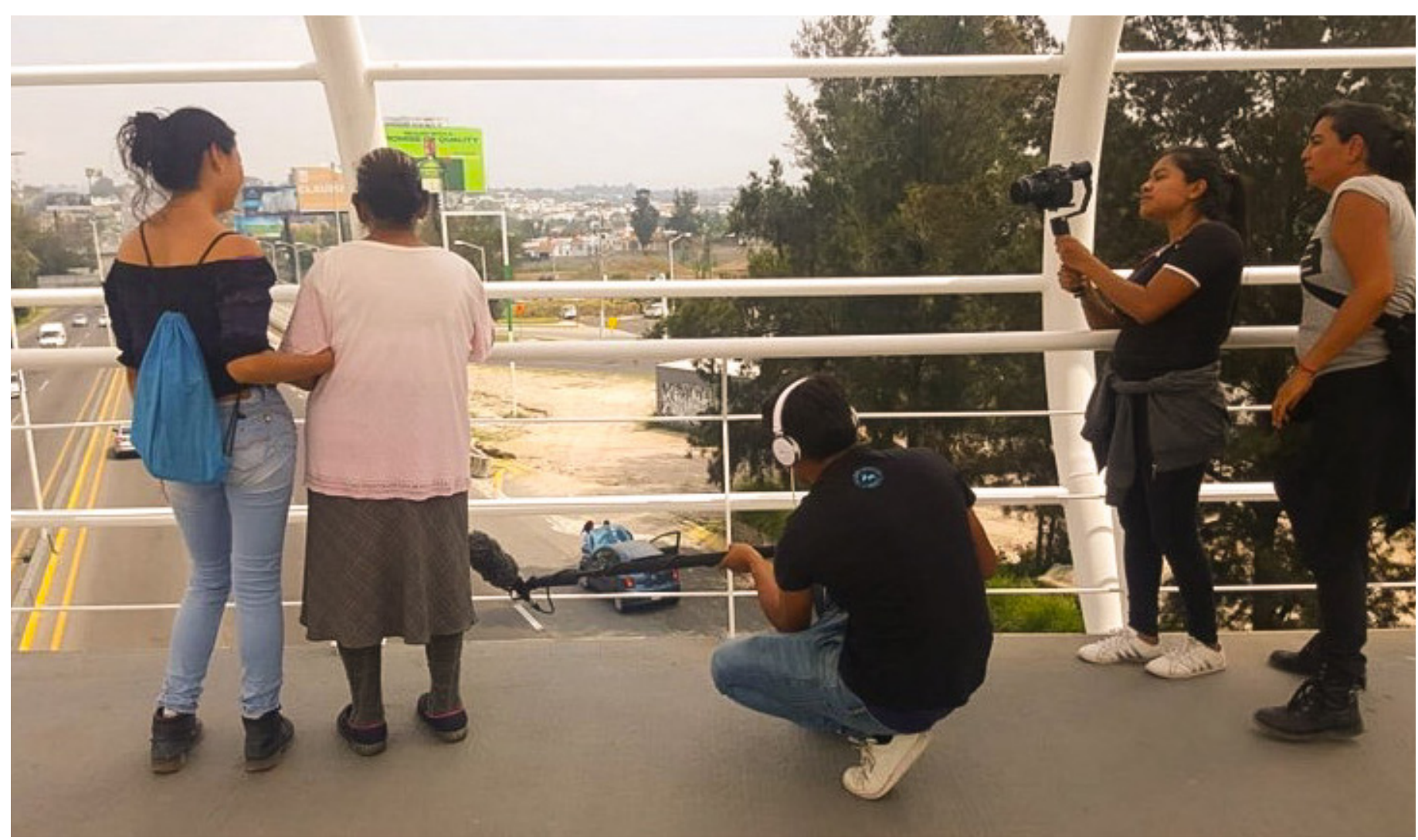

Figura 2. Taller de cine comunitario. Fuente: elaboración propia. 


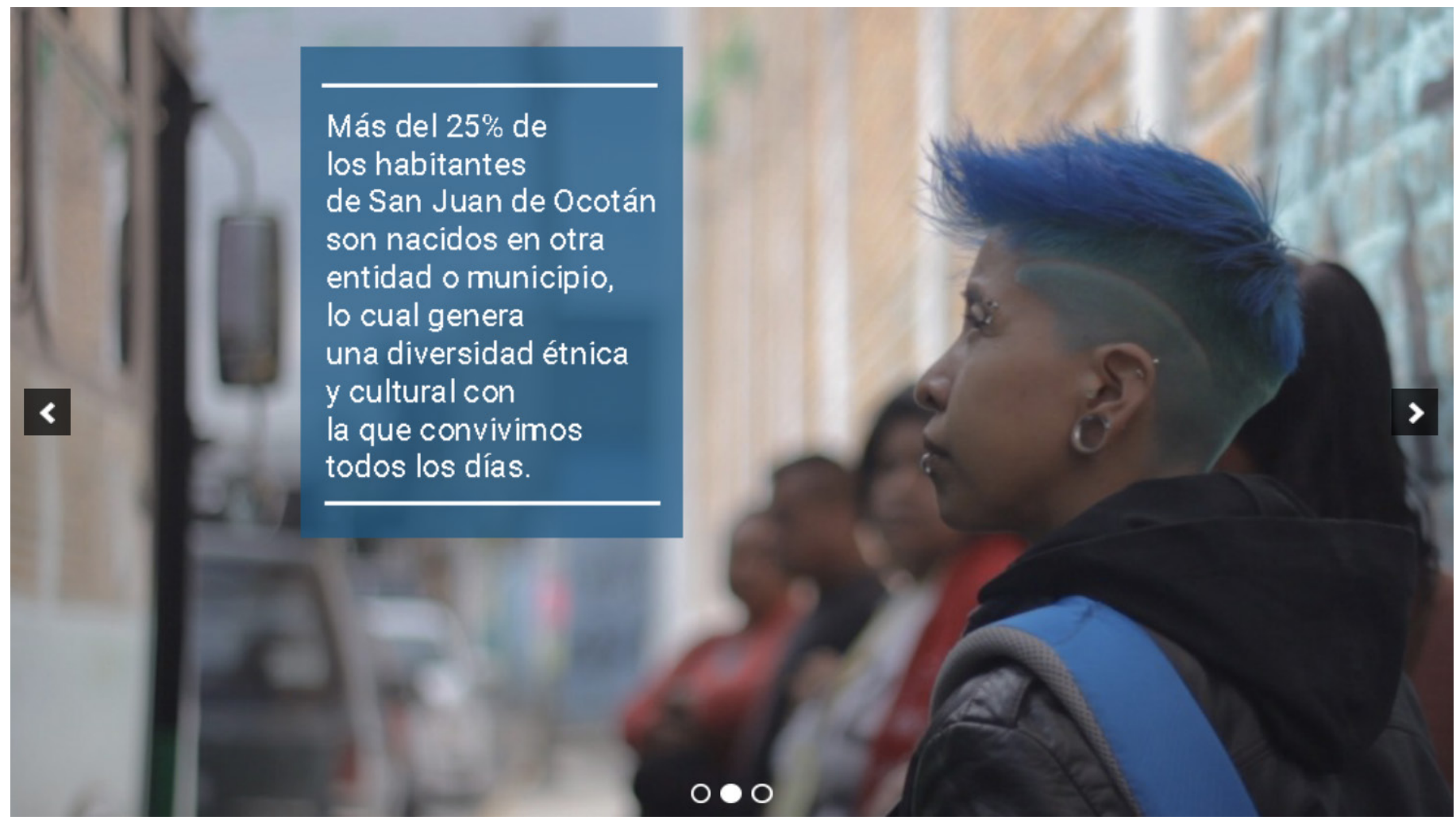

Figura 3. Documental Como las aves. Fuente: elaboración propia.

Los productos emanados del Taller de cine comunitario se agruparon de la siguiente manera en el documental web:

- Quiénes somos. En este apartado se muestran los autoretratos realizados durante el Taller, en un ejercicio donde uno de los chicos dirigía su autorretrato y posaba, mientras otro de ellos tomaba la fotografía.

- Videomapa. En el videomapa se observan los siguientes ejercicios:

1. Lugares. Estas son una serie de películas breves, de menos de 1 minuto, inspiradas en las películas de un solo plano, sin movimientos de cámara, realizadas en los orígenes del cine. Este fue un ejercicio individual y en ellas se observan los lugares que fueran más significativos del pueblo, para ellos. En este ejercicio se trabajó también el diseño sonoro.

2. Caminos. El ejercicio consistió en una serie de movimientos de cámara sobre trayectos que cada uno de los jóvenes solían hacer dentro del pueblo. Estos debían mostrarse en un solo plano.

3. Vida cotidiana. Incluye tres cortometrajes de máximo 3 minutos de duración sobre situaciones cotidianas del pueblo. Este ejercicio se realizó en equipos y consistió en la grabación de una acción en varios planos que posteriormente se editaron.
- Nuestros abuelos. Este apartado incluye una serie de podcast y de ensayos fotográficos sobre algunos de los adultos mayores del pueblo. Primeramente los jóvenes escucharon algunas entrevistas realizadas a los adultos mayores del pueblo y utilizando esos audios diseñaron un ensayo fotográfico. Posteriormente también editaron los audios de las entrevistas en forma de podcast para el documental web.

- Los tastuanes. Consiste en una serie de cortometrajes de 3 a 5 minutos sobre su experiencia personal de la Fiesta de los tastuanes. Este fue un ejercicio individual basado en un guion con edición de imagen y de audio.

- Historias. En este apartado se encuentran los tres documentales finales del Taller de cine comunitario de 30 minutos de duración aproximadamente.

1. El primero de los documentales finales se titula Como las aves (Figura 3), y está dedicado a los sectores de migrantes, principalmente zapotecos que viven en el pueblo. En su sinopsis se puede leer: "En San Juan de Ocotán existe una diversidad de lenguas, gastronomía y tradiciones, gracias a la migración". En la segunda parte del trabajo, para el documental web, se realizó un fotoreportaje sobre la comida que los migrantes han traído a San Juan de Ocotán.

2. El segundo de ellos, se titula Un kilo de manzanas amarillas (Figura 4), el cual aborda la relación entre la música, el baile, el amor y la sexualidad en San Juan de Ocotán y en cuya sinopsis se puede leer: "Las relaciones amorosas, 


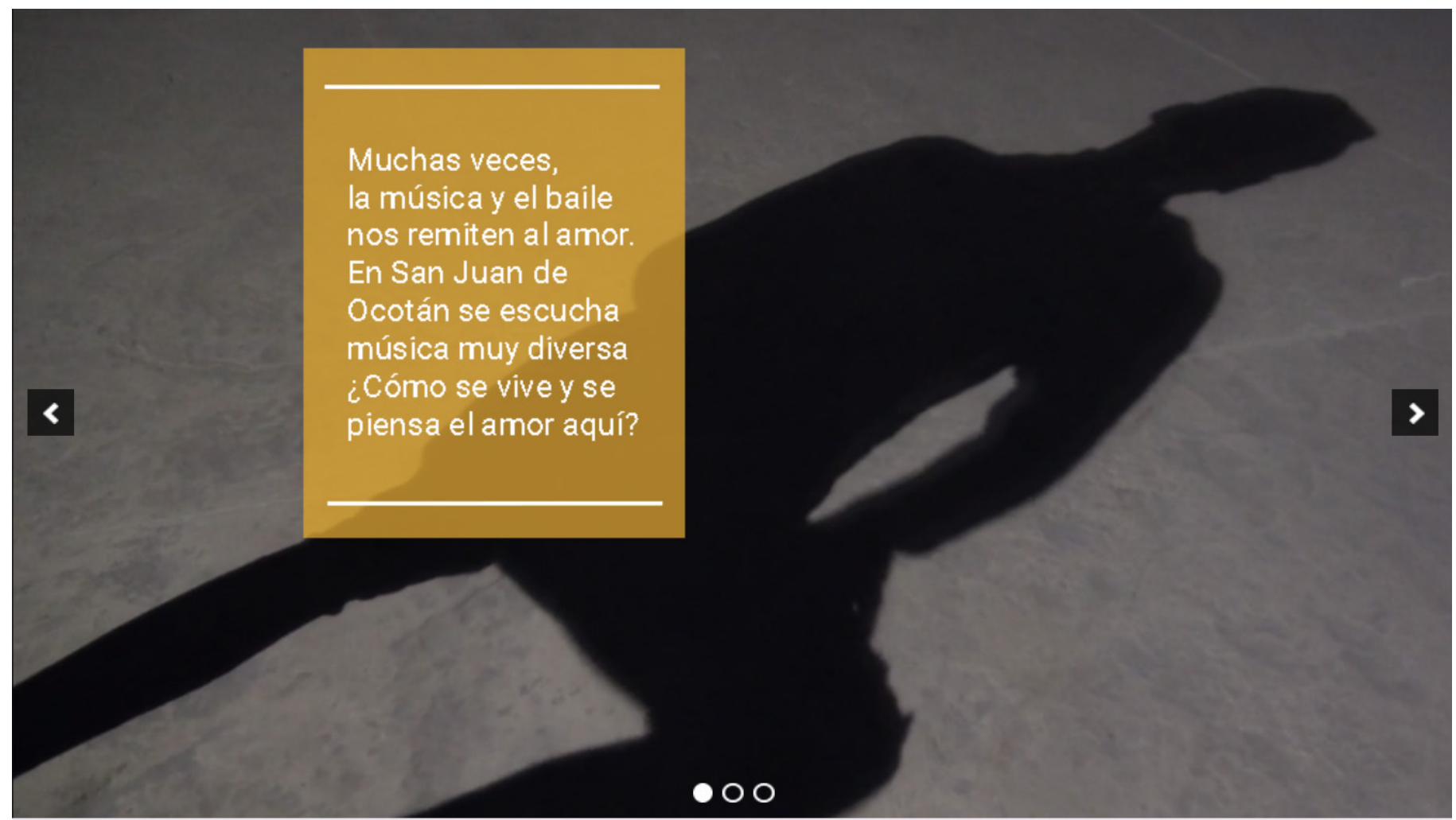

Figura 4. Documental Un kilo de manzanas amarillas. Fuente: elaboración propia.

al ritmo de SJO son más divertidas". En la segunda parte del trabajo, para el documental web, se realizó un fotoreportaje y una pista de audio sobre los músicos del pueblo.

3. El tercero se titula Propiedad privada (Figura 5), el cual aborda la transformación que San Juan de Ocotán ha sufrido en los últimos años a consecuencia de la venta de la tierra, el crecimiento de la ciudad y la presión inmobiliaria. En su sinopsis se puede leer: "Antiguamente nuestro pueblo era campesino. La pobreza y el crecimiento de la ciudad hizo que la mayoría de nuestros abuelos vendiera su tierra". En la segunda parte del trabajo, para el documental web, se acompañó con una línea de tiempo sobre la tenencia de la tierra en San Juan de Ocotán, titulada Historia de nuestra tierra, un mapa interactivo basado en Google Earth en el que se puede observar cómo ha cambiado el territorio de 1984 a 2019, así como el mapa de la ampliación del ejido de 1934.

Una vez concluido el Taller, se llevó a cabo una presentación en la plaza del pueblo. Al finalizar esta etapa de trabajo, la mayoría de los jóvenes participantes del Taller decidieron continuar el proceso de creación colaborativa y se conformaron en una productora comunitaria autonombrada Documachete: productora en resistencia de San Juan de Ocotan ${ }^{3}$. Con ellos se inició un nuevo proceso de trabajo para crear un documental web que permitiera acercar al resto de habitantes del pueblo todo el material producido durante el Taller en forma de un acervo de memoria barrial.

Para el documental web se decidió utilizar recursos que pudieran ser de acceso libre, no sólo porque el proyecto tenía presupuesto limitado, sino porque se buscaba que estos se convirtieran en herramientas que los chicos pudieran seguir utilizando. El documental web se organizó en torno a los productos realizados durante el Taller y a partir de allí se realizaron algunos productos más, mismos que complementaron la experiencia y facilitaron la navegación, como una línea de tiempo sobre la tenencia de la tierra en San Juan de Ocotán, la cual acompañara al documental Propiedad privada; la edición en forma de podcast de algunas entrevista a adultos mayores del pueblo y que se escuchan mientras se ven sus retratos; una pieza de arte sonoro y un reportaje fotográfico sobre los músicos del pueblo, los cuales acompañan el apartado del documental de Un kilo de manzanas amarillas; y por último, un reportaje sobre la comida que venden los migrantes que han llegado a San Juan de Ocotán, para acompañar el documental Como las aves.

\section{Resultados y conclusiones}

El primer elemento a recalcar es que los resultados de un proyecto como este se puede observar tanto en las prácticas y los procesos como las evidencias físicas o los productos generados por el mismo. Es decir, el proceso fue tan importante como los productos generados en él. Podríamos decir, al seguir en sentido inverso del recorrido de la producción de contenidos, 


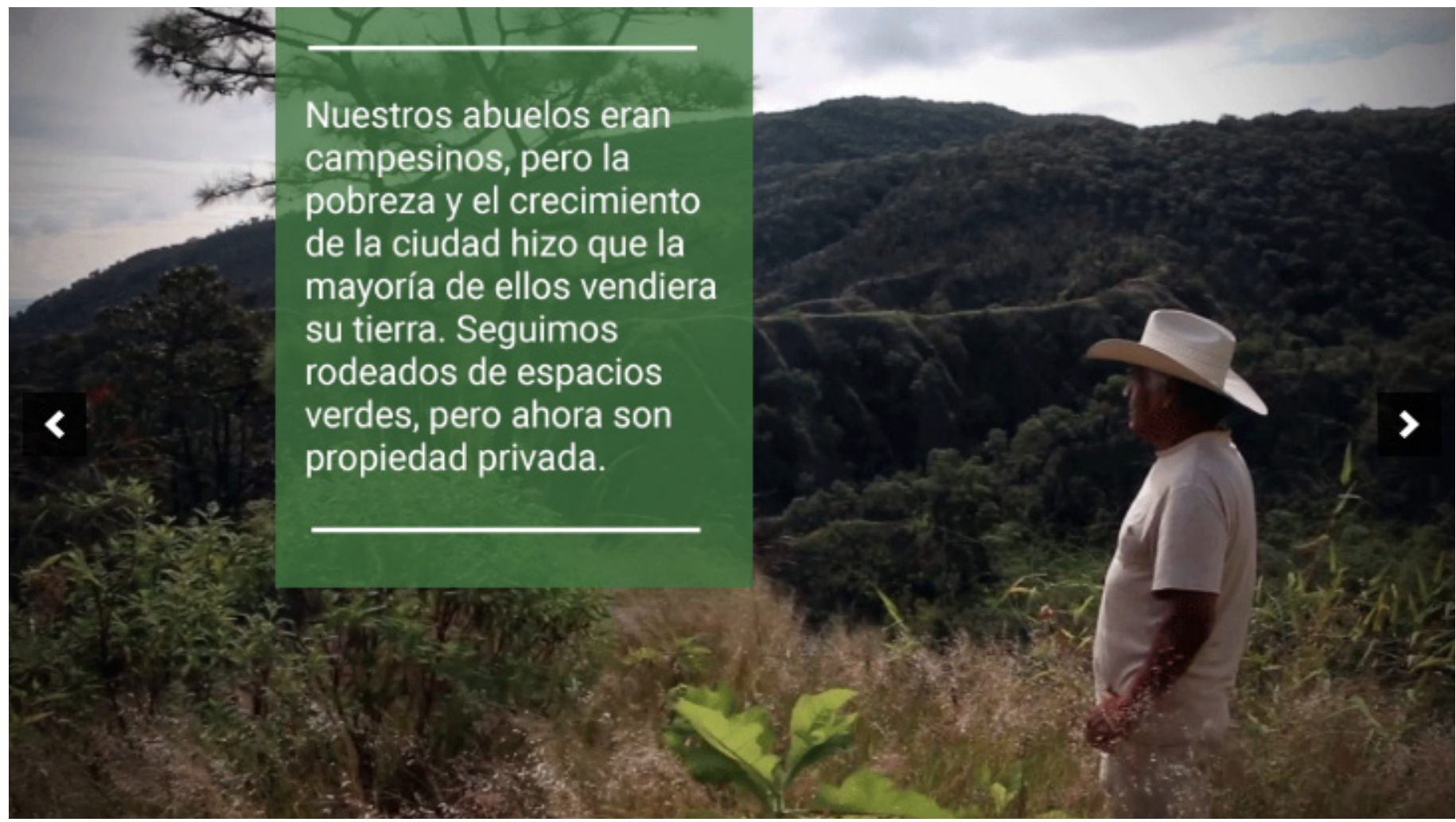

Figura 5. Documental Propiedad privada. Fuente: elaboración propia.

que en realidad, la conformación del colectivo y el establecimiento de las reglas de producción (asamblearia, participativa, horizontal) fue uno de los productos más importantes del proyecto.

Una de las críticas que se suele hacer a los viejos medios de comunicación y su lógica broadcast, es su unidireccionalidad, ya que la dinámica de circulación de mensajes suele ser de "uno a muchos", a diferencia de los medios digitales interactivos cuya direccionalidad es de "muchos a muchos", puesto que en ellos los usuarios no sólo consumen los mensajes, sino que también tienen la posibilidad de producirlos. Mientras que en los viejos medios, la interacción del usuario y la posibilidad de decidir el contenido y la forma de los mensajes es prácticamente nula, en los nuevos medios, la interacción y la participación de los usuarios es esencial (Scolari, 2008). Una de las apuestas de este proyecto fue el colocar en el mapa de la discusión una tercera opción, y para ello, fracturar la dimensión unidireccional del proceso de producción y circulación de productos audiovisuales a través de procesos colaborativos entre profesionales del audiovisual y los posibles consumidores, en los procesos de creación, producción y circulación de los mismos. Por eso nos interesa la visión de la producción participativa como parte de la integración de habilidades transmediales y de la generación de memoria transmedia en el sentido de Erll y Seydel.

El trabajo realizado en San Juan de Ocotán permite descentrar tanto la figura institucional de los medios de comunicación, como la de productor/usuario individual de los medios inte- ractivos, y pensar en una figura de producción colectiva entre productores y usuarios, que a su vez está desligada a la producción mediática institucional y pensada desde una lógica distinta y anclada en la memoria cultural.

La situación de brecha escolar y tecnológica de la mayoría de miembros del colectivo no significó falta de imaginación social o de posibilidad de creación de contenidos, como lo demostró la producción sistemática de videos, fotografías, entrevistas y páginas web. Es en este ejercicio de conformación del grupo de trabajo donde se da la posibilidad de trabajar junto con ellos los proyectos, inquietudes y temáticas que responden a su experiencia personal de su barrio y la realidad que viven cotidianamente.

En una tercera fase de trabajo, Documachete tomó el control de la producción y decidió continuar trabajando de forma colaborativa un documental sobre la Fiesta de los tastuanes. En esta última fase de trabajo, se ha procurado que los jóvenes comiencen a adquirir habilidades en la gestión y administración financiera de la producción mediática y cinematográfica, en los mecanismos y hábitos de los sistemas de fondos para proyectos culturales en México. Para ello, se trabajó en colaboración con ellos para la aplicación de algunos fondos de cultura y de cine. Algunos de ellos se consiguieron y actualmente el documental está en fase de desarrollo. Aquí se reveló otro de los mecanismos de exclusión, pues la producción de contenidos parecía la forma natural para intentar disminuir la brecha tecnológica y social, pero la gestión y acceso a redes de generación de sentido se mostró como una forma de mar- 
ginación mayor, puesto que los guiones y las carpetas que las instituciones piden para financiar los proyectos también se exigen en forma escrita. En ese sentido el trabajo colaborativo junto con profesionales cómplices como el colectivo Documotora se ha vuelto crucial para el acompañamiento de estos procesos.

Para dar cuenta de los procesos de exclusión que genera la escuela, el sociólogo francés Pièrre Bourdieu (1997) evoca la termodinámica, en la que pareciera que hay un demonio entre las partículas, el cual calienta a las más rápidas y enfría a las más frías, generando cada vez más diferencia de temperatura entre ellas. En el contexto postcolonial de Latinoamérica, uno de estos demonios es la priorización del conocimiento logocéntrico sustentado en la razón, y la lectoescritura, lo cual se vuelve un sistema de exclusión del conocimiento de aquellos pueblos cuya matriz de conocimiento es distinta. Para combatir ese demonio, podemos afirmar que hay tres tipos de prácticas que debemos o queremos considerar como parte del proceso de alfabetidad transmedial: creación de comunidades y de redes de colaboración en las que los jóvenes no se enfrentan a los procesos de manera individual, sino acompañada, tanto por profesionales como por sus propios pares, prácticas disciplinares o laborales orientadas a la producción de contenidos que se construyan como espacios de expresión y de habla que permita dar cuenta de su propia forma de ver el mundo, y finalmente, la participación dentro del sistema de generación de cultura específico, adecuada a su propia matriz de conocimiento, que la puedan vivir como un espacio lúdico y creativo en vez de un espacio de exclusión.

Esta última parte se muestra como un ejercicio prioritario de fractura de las inercias de exclusión de jóvenes de barrios como San Juan de Ocotán. Y al ser aplicado, nuestro proyecto en un espacio de educación no formal, se muestra la relevancia de la contextualización de las acciones y de la conformación de la comunidad como principio relacional que posibilita la producción. Las habilidades y alfabetidades se muestran de manera evidente, pero pareciera haber mucho más énfasis en las capacidades de conectar y aplicar a situaciones específicas lo aprendido. Ahí está el reto y el objetivo a seguir.

\section{Notas al final}

1. Disputas infrapolíticas por la mirada. Autorrepresentaciones audiovisuales de los jóvenes del pueblo urbano de San Juan de Ocotán, Jalisco. Tesis de Doctorado en Ciencias Antropolígicas, por la Universidad Autónoma de México (UAM).

2. Facebook de Documotora: https://www.facebook.com/Documotora/

3. Facebook de Documachete: https://www.facebook.com/Documachete

\section{Bibliografía}

Artz, L. (2020) A political economy for social movements and revolution: popular media access, power, and cultural hegemony. Third World Quarterly, 47(8), 1388-1405, https://doi.org/10.1080/01436597.

\subsection{5}

Austin, J. L. (2020). How To Do Things with Words. Barakaldo Books.

Bourdieu, P. (1997). Razones prácticas. Sobre la teoría de la acción. Anagrama.

Colmenares, A.M. (2012): Investigación-acción participativa: una metodología integradora del conocimiento y la acción. Voces y Silencios: Revista Latinoamericana de Educación, 3(1), 102-115.

Dedemaine, A., Marcoux, G. y St. Amand, I. (2020). Indigenous Cinema and Media in the Americas: Storytelling, Communities, and Sovereignties. Canadian Journal of Film Studies / Revue Canadienne D'études Cinématographiques 29(1), pp 27-51. https://doi.org/10.3138/ cjfs.29.1.02

Erll, A. (2008). Literature, Film, and the Mediality of Cultural Memory. En A. Erll y A. Nünning (eds.) A Companion to Cultural Memory Studies, (pp. 389-398). Walter de Gruyter.

Gumucio Dagron, A. (2014). El cine comunitario en América Latina y el Caribe. FNCL.

Jenkins, H. (2008). Convergence culture: La cultura de la convergencia de los medios de comunicación, Paidós.

Johnson, D. (2013). A History of Transmedia Entertainment. Spreadable Media: Creating Value and Meaning in a Networked Culture. http://spreadablemedia.org/essays/johnson/\#.YZKRSb2ZMqv

McCully, B. T. (1940). English Education and the Origins of Indian Nationalism. Columbia University Press.

Mejía Lara, A.C. (2017). Un pan y un café. El documental colaborativo de DocuPerú como contrapunto del modelo hegemónico de producción cinematográfica. En C. S. Gordillo y A. C. Mejía (eds.), Miradas urgentes: Sujetos, estéticas y memorias del documental latinoamericano contemporáneo, (pp.s/n), Editorial Foc.

Mejía Lara, A.C. (2013). Siempre fuimos guerreros. Tácticas y estrategias simbólicas de los habitantes de San Juan de Ocotán (Tesis de maestría). Guadalajara: Instituto Tecnológico y de Estudios Superioes de Occidente.

\section{Mittell, J. (2015). Complex TV. NYU Press.}

Piscitelli, A., Adaime, I.y Binder, I. (comps.) (2010). El "Proyecto Facebook" y la posuniversidad. Sistemas operativos sociales y Entornos abiertos de aprendizaje. Ariel/Fundación Telefónica.

Ravenscroft, A. (2020) Participatory internet radio (RadioActive101) as a social innovation and co-production methodology for engagement and non-formal learning amongst socially excluded young people, International Journal of Inclusive Education, 24(9), 997-1018, https://doi.org/10.1080/13603116.2019.1700312

Scolari, C. A. (2017). Transmedia Literacy. https://transmedialiteracy. org/

Scolari, C. A. (2013). Narrativas transmedia. Cuando todos los medios cuentan. Deusto

Scolari, C. A. (2008). Hipermediaciones. Elementos para una Teoría de la Comunicación Digital Interactiva. Gedisa.

Searle, J. (1969). Speech Acts. An Essay in the Philosophy of Language. Cambridge University Press.

Seydel, U. (2018). La memoria cultural, su dinamismo y sus conflictos. Alternativas, (9), 1-31. https://alternativas.osu.edu/es/issues/autumnspring-9-2018-19/essays6/intro2.html

Spivak, G. C. (1997). Puede hablar el sujeto subalterno?. Orbis 
Tertius, 3(6), 1-44. https://www.orbistertius.unlp.edu.ar/article/view/ OTv03n06t01

Tamayo Gómez, C.; Freeman, M. y Morales Velásquez, E. (eds.) (2018). Arqueología transmedia en América Latina: mestizajes, identidades y convergencias. EAFIT.

Wyatt, S., Bier, J., Harris, A. y van Heur, B. (2013). Participatory Knowledge Production 2.0: Critical Views and Experiences, Information. Communication \& Society, 16(2), 153-159. https://doi.org/10.1080/136 9118X.2012.746382

Zavala y Leetoy (2016). Documental participativo como herramienta de agencia cultural: El Salto, un caso de estudio. IC Revista científica de información y comunicación, (13), 235-261. http://hdl.handle. net/11441/68279

\section{Páginas web citadas}

- Web del taller de cine comunitario: https://somossanjuandeocotan.org

- Apartado Quiénes somos: https://somossanjuandeocotan. org/quienes-somos

- Apartado Videomapa: https://somossanjuandeocotan.org/ videomapa

- Apartado Nuestros abuelos: https://somossanjuandeocotan.org/nuestros-abuelos

- Apartado Los tastuanes: https://somossanjuandeocotan. org/los-tastuanes

- Apartado Historias: https://somossanjuandeocotan.org/ historias

- Documental Como las aves: https://somossanjuandeocotan.org/como-las-aves-2

- Documental Un kilo de manzanas amarillas: https://somossanjuandeocotan.org/un-kilo-de-manzanas-amarillas

- Documental Propiedad privada: https://somossanjuandeocotan.org/propiedad-privada

\section{CV}

Afra Citlalli Mejía Lara. Doctora en Antropología por la Universidad Autónoma Metropolitana (México). Máster en Cine Documental de Creación por la Universitat Pompeu Fabra y Maestría en Comunicación de la Ciencia y la Cultura por el ITESO (México). Es miembro fundador de la Red de Estudios Visuales Latinoamericanos (REVLAT) y de los Documentalistas Mexicanos en Red (DOC-RED).

Diego Zavala Scherer. Doctor en Comunicación Social por la Universidad Pompeu Fabra. Es Profesor Investigador de la Escuela de Humanidades y Educación del Tecnológico de Monterrey. Miembro de la Cátedra en Narrativas Tansmedia de la Universidad de Rosario, Argentina y del Grupo I+D+C Museum de la Universidad Complutense de Madrid, España. Director de Virtualis. Revista de Cultura Digital del Tecnológico de Monterrey. Coeditor del libro Cine político en México (1968-2017).

\section{осм Observatorio deCibermedios}

El Observatorio de Cibermedios es una producción del Grupo de Investigación en Documentación Digital y Comunicación Interactiva (DigiDoc) del Departamento de Comunicación de la Universitat Pompeu Fabra.

El Observatorio de Cibermedios (OCM) forma parte del proyecto del Plan Nacional "Narración interactiva y visibilidad digital en el documental interactivo y el periodismo estructurado". RTI2018-095714-B-C21 (MINECO/FEDER), Ministerio de Ciencia, Innovación y Universidades (España). 